\title{
Use of dual-grating sensors formed by different types of fiber Bragg gratings for simultaneous temperature and strain measurements
}

\author{
Xuewen Shu, Donghui Zhao, Lin Zhang, and Ian Bennion
}

\begin{abstract}
We report on a systematic investigation of the dependence of both temperature and strain sensitivities on the fiber Bragg grating type, including the well-known Type I, Type IIA, and a new type that we have designated Type IA, using both hydrogen-free and hydrogenated B/Ge codoped fibres. We have identified distinct sensitivity characteristics for each grating type, and we have used them to implement a novel dual-grating, dual-parameter sensor device. Three dual-grating sensing schemes with different combinations of grating type have been constructed and compared, and that of a Type IA-Type IIA combination exhibits the best performance, which is also superior to that of previously reported gratingbased structures. The characteristics of the measurement errors in such dual-grating sensor systems is also presented in detail. (C) 2004 Optical Society of America
\end{abstract}

OCIS codes: $060.2370,060.2310$.

\section{Introduction}

UV-induced fiber Bragg gratings (FBGs) have attracted great attention in recent years because of their potential use in a wide range of applications, particularly in monitoring the structural integrity of buildings and bridges and in the aerospace industry, where monitoring the fatigue loading of advanced composite materials is essential. ${ }^{1-3}$ The distinct advantages of the FBG include providing absolute wavelength encoding of the information and wavelength division multiplexing capabilities. However, the simultaneous sensitivity to temperature and strain of the resonant wavelength of the FBG has complicated independent measurement procedures for these two measurands. For solution of the problem of cross sensitivity, several methods have been proposed and investigated, including dualwavelength superimposed gratings, ${ }^{2}$ the use of firstand second-order diffraction from a single $F B G,{ }^{3}$ a hybrid FBG/long period grating (LPG), ${ }^{4}$ a FBG superimposed on a polarization-rocking filter, 5 and

The authors are with the Photonics Research Group, Aston University, Birmingham, B4 7ET, United Kingdom. X. Shu's e-mail address isx.shu@aston.ac.uk.

Received 30 September 2003; revised manuscript received 16 December 2003; accepted 22 January 2004.

0003-6935/04/102006-07\$15.00/0

(C) 2004 Optical Society of America spliced FBGs inscribed in different fibers., ${ }^{6,7}$ All these methods are, in effect, dual-grating methods, and although they have achieved parameter discrimination, most of them are far from suitable for practical use owing to either insufficiently large differentiation of the thermal and strain coefficients between the gratings or overcomplex signal interrogation and processing requirements. Additionally, in the case of the otherwise-attractive FBG-LPG structures, the broad spectral response of the LPG imposes severe limitations on multiplexing.

Recently, we reported a new FBG type (Type IA) with a significant low-temperature coefficient and demonstrated its application for effective simultaneous temperature and strain measurements. ${ }^{8,9}$ In this paper, we report on the detailed results of a systematic investigation of the dependence of both temperature and strain sensitivities on the grating type, including Type I, Type IIA, and Type IA, using both hydrogen-free and hydrogenated $\mathrm{B} / \mathrm{Ge}$ codoped fibers. We have identified distinct sensitivity characteristics for each grating type, and we have used them to implement a dual-grating, dual-parameter sensor device with performance superior to that of previously reported grating-based structures. ${ }^{2-7}$

The outline of this paper is as follows. In Section 2 , we show the characteristics of different grating types generated in $\mathrm{B} / \mathrm{Ge}$ codoped fibers, which include their distinct growth behaviors and their temperature and strain properties. In Section 3, we 

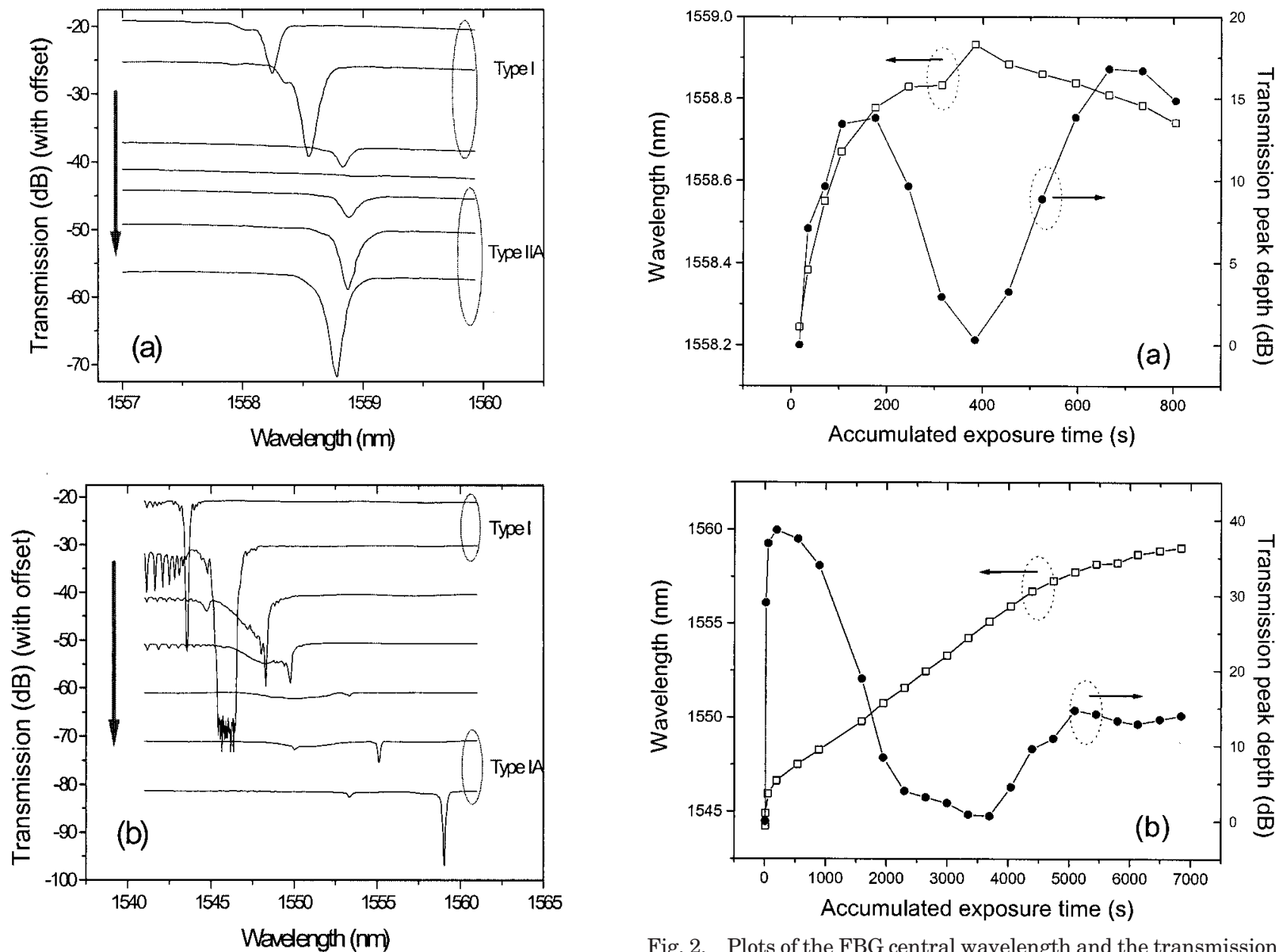

Fig. 1. Evolution of the transmission spectrum of a grating inscribed in (a) a hydrogen-free and (b) a hydrogenated B/Ge codoped fiber with long UV exposure. The arrows indicate the direction of increasing exposure time.

compare the performance of different combination schemes of dual-grating sensors formed by different grating types. In Section 4, we present error analysis for such dual-grating sensor systems. Section 5 concludes the paper.

\section{Charcteristics of Different Grating Types Generated in B/GE Codoped Fiber}

\section{A. Growth Behavior of Different Grating Types}

The FBGs used in these investigations were UVinscribed in $\mathrm{B} / \mathrm{Ge}$ codoped fibers, with and without hydrogen-loading, using a frequency-doubled argon laser at a 244-nm wavelength and a phase mask. The spectral profiles of the gratings were measured in situ during UV exposure by use of a broadband LED and an optical spectrum analyzer with 0.1-nm resolution. During exposure, quite different growth characteristics were observed for gratings inscribed in hydrogen-free and hydrogenated fibers, and transformations from one type to another occurred with long UV exposures. For the hydrogen-free fiber, as shown in Fig. 1(a), a Type I grating (normal) was first

Fig. 2. Plots of the FBG central wavelength and the transmission peak depth as a function of accumulated exposure time. (a) and (b) correspond to (a) and (b) in Fig. 1, respectively.

formed, grew to a maximum strength as the exposure proceeded, and then grew weaker to the point of complete erasure, with the Bragg wavelength shifting continuously to longer wavelengths throughout the exposure. With further UV exposure, a Type IIA (abnormal) grating ${ }^{10,11}$ was regenerated in the fiber, growing stronger with increasing exposure and with the Bragg wavelength now shifting continuously to shorter wavelengths. This behavior was previously observed and characterized. ${ }^{8,9}$ However, with the hydrogenated fiber, a quite different behavior was observed following the formation and erasure of the initial Type I grating: As shown in Fig. 1(b), grating regeneration occurred with increasing exposure, but the Bragg wavelength shift in this case was continuously to longer wavelengths, in marked contrast with Type IIA grating growth in hydrogen-free fiber. The regenerated abnormal grating has been designated Type IA (Ref. 10). As can be seen in Fig. 1(b), the magnitude of the Type IA redshift is significantly larger than the blueshift accompanying Type IIA grating formation, and a 16-nm shift was recorded after $2 \mathrm{~h}$ of UV exposure. Figures 2(a) and 2(b) depict the wavelength shift and the transmission peak depth as a function of accumulated exposure time 

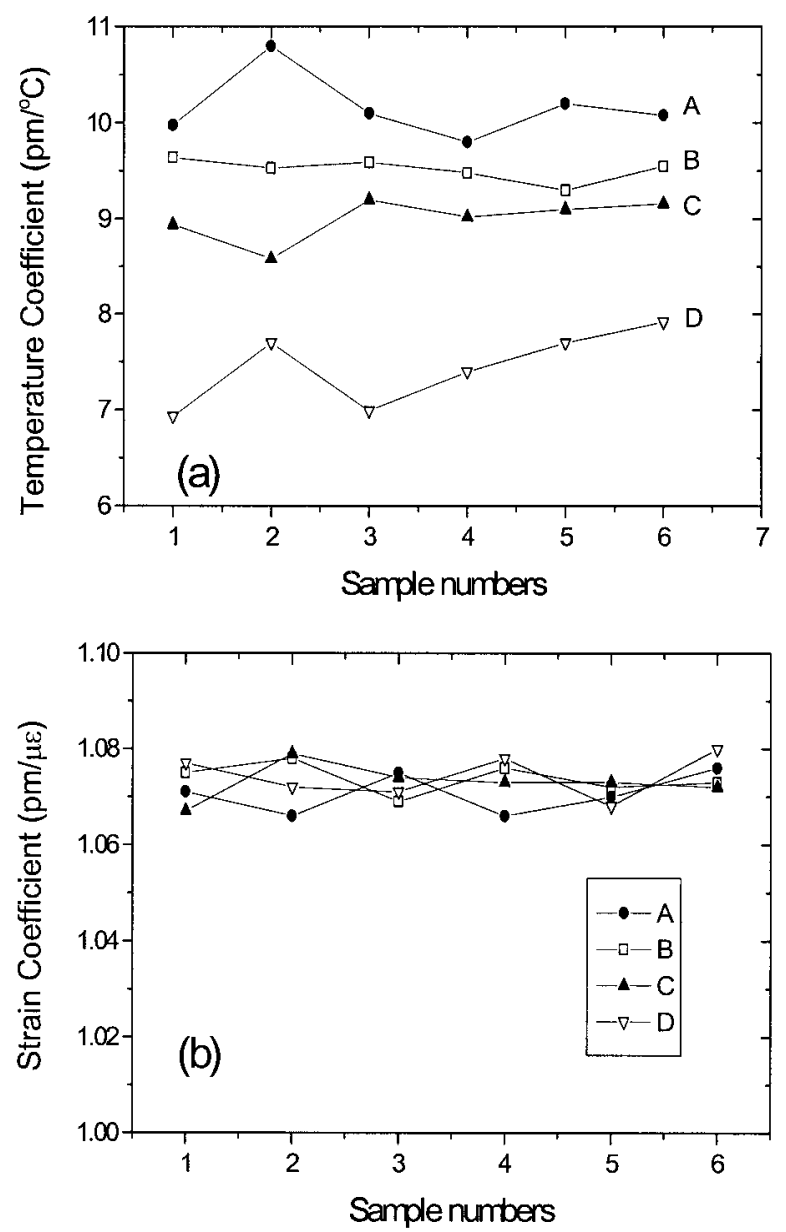

Fig. 3. Distribution of (a) the measured temperature coefficient and (b) the strain coefficient for different types of FBG inscribed in B/Ge codoped fiber; A, Type IIA gratings; B, Type I gratings in hydrogen-free fiber; C, Type I gratings in hydrogenated fiber; D, Type IA gratings.

corresponding to the grating evolutions in Figs. 1(a) and 1(b). Comparing Figs. 2(a) and 2(b), we can clearly see that the speed of conversion from a Type I grating to a Type IIA grating in hydrogen-free fiber is almost ten times faster than that from a Type I grating to a Type IA grating in hydrogenated fiber.

For all three types of grating, we subsequently examined the spectral profiles following a period of annealing at $95{ }^{\circ} \mathrm{C}$. In all cases, blueshifts were recorded, but the $3-5-\mathrm{nm}$ shifts obtained from Type IA gratings were far larger than the $<1 \mathrm{~nm}$ measured for the Type I and Type IIA gratings.

\section{B. Dependence of Temperature and Strain Coefficients on FBG Type}

For a comparative study of the temperature and strain coefficients, we produced 24 gratings, six of each type-two batches of Type I gratings were included, one in hydrogen-free and the other in hydrogenated fiber. We measured the thermal response of each FBG by heating it from $0{ }^{\circ} \mathrm{C}$ to $80{ }^{\circ} \mathrm{C}$ in incremental steps of $10^{\circ} \mathrm{C}$ using a thermoelectric cooler. The thermal coefficients measured are plot-

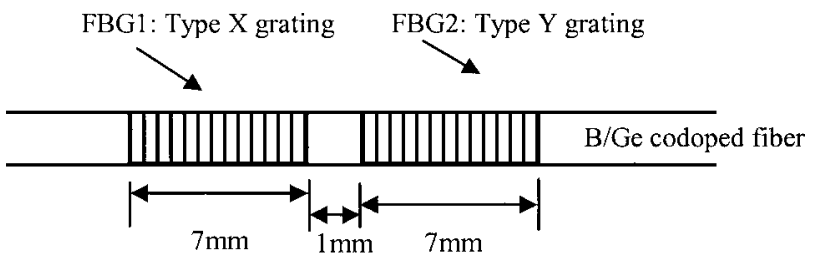

Fig. 4. Schematic of the proposed dual-grating sensor employing concatenated different-type gratings.

ted in Fig. 3(a) for all 24 gratings; the values obtained in hydrogen-free fibers [(A) and (B)] are higher than those in hydrogenated fibers [(C) and (D)]. For Type I gratings, the average value is $\sim 6 \%$ different. Among the four sets of gratings, Type IIA yields the highest temperature coefficient, $10.80 \mathrm{pm} /{ }^{\circ} \mathrm{C}$, whereas Type IA exhibits the lowest at $6.93 \mathrm{pm} /{ }^{\circ} \mathrm{C}$. The 55\% difference between these two is substantially larger than in all previously reported dualgrating sensors.

We also examined the strain response for each type of grating by stretching the fibers between two translation stages and monitoring the Bragg wavelength shift. The strain coefficients measured are plotted in Fig. 3(b) for all 24 gratings; it was found that the gratings showed a similar strain sensitivity, regardless of grating type. The underlying mechanisms responsible for the dissimilar thermal, and similar strain responses are not considered here.

\section{Dual-grating Sensors for Temperature-Strain Discrimination}

The results presented in Section 2 can be exploited to conceive dual-grating-based sensors for temperature and strain, with significant performance advantages over the alternatives previously reported. Since gratings of each type studied exhibit narrow spectral (Bragg) response features, and grating of different types may be readily produced in a single fiber, such dual-grating sensors can take full advantage of FBG wavelength multiplexing capabilities.

We propose here a dual-grating sensing scheme by inscribing two different types of grating in adjacent sections of a single fiber, as illustrated in Fig. 4. Owing to the fact that four types of grating can be fabricated in either hydrogenated fiber or hydrogenfree fiber, one can use any two different types to construct a dual-grating sensor, which theoretically results in six different combinations, as shown in Table 1.

Table 1. Combination of Two Different Types of Grating in a Dual-Grating Sensing Scheme

\begin{tabular}{lcccc}
\hline \multicolumn{1}{c}{ Grating Type } & $\begin{array}{c}\text { Type I } \\
\text { without H }\end{array}$ & $\begin{array}{c}\text { Type I } \\
\text { with H }\end{array}$ & Type IA & Type IIA \\
\hline Type I without H & No & Yes & Yes & Yes \\
Type I with H & Yes & No & Yes & Yes \\
Type IA & Yes & Yes & No & Yes \\
Type IIA & Yes & Yes & Yes & No \\
\hline
\end{tabular}



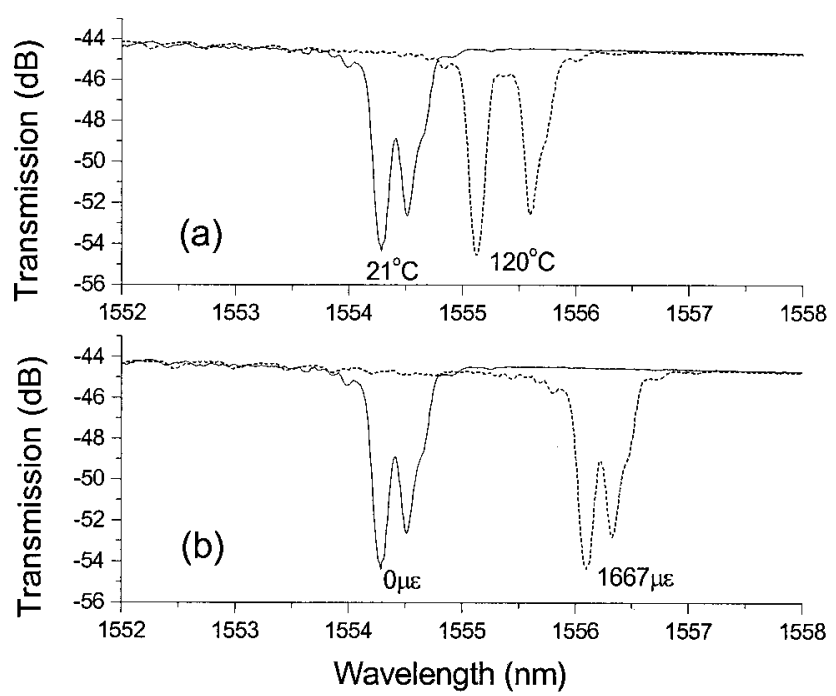

Fig. 5. Spectral response changes in the first dual-grating sensor induced by (a) temperature and (b) strain.

Experimentally, we have chosen three combinations to investigate such dual-grating sensors. The first dual-grating sensor is the combination of Type IA grating and Type IIA grating. The Type IA grating, with a length of $7 \mathrm{~mm}$, was first inscribed in a length of hydrogen-loaded B/Ge fiber. The fiber was annealed for $24 \mathrm{~h}$ at $95^{\circ} \mathrm{C}$ to stabilize the grating properties and remove residual hydrogen. The Type IIA grating, with the same length as the Type IA structure but with a slightly different center wavelength, was then inscribed in the fiber separated from the Type IA grating by $1 \mathrm{~mm}$. The fiber was annealed once more for $48 \mathrm{~h}$ at $120^{\circ} \mathrm{C}$ to stabilize the final structure.

The transmission spectrum of the unstrained dualgrating sensor at room temperature $\left(21^{\circ} \mathrm{C}\right)$ is shown by the solid curve in Fig. 5. The peak wavelength separation between the constituent gratings is 0.22 $\mathrm{nm}$. When the structure was raised to a temperature of $120^{\circ} \mathrm{C}$, the separation between the peaks increased significantly, as shown in Fig. 5(a). When, on the other hand, strain was applied at room temperature, the peak separation remained essentially unchanged, as shown in Fig. 5(b). We investigated the linearity of the thermal response by heating the sensor from $0{ }^{\circ} \mathrm{C}$ to $80{ }^{\circ} \mathrm{C}$ and measuring the wavelength shifts for the two peaks; the results are given in Fig. 6(a). It is clear that the thermal response of each grating type is linear, but there is a substantial difference between the respective temperature coefficients. From the best-fit straight lines in Fig. 6(a), we obtain coefficients of 7.37 $\mathrm{pm} /{ }^{\circ} \mathrm{C}$ for the Type IA grating and $10.02 \mathrm{pm} /{ }^{\circ} \mathrm{C}$ for the Type IIA; the difference between the two is $\sim 36 \%$. Following thermal evaluation, the structure was strained over the range $0-2000 \mu \varepsilon$, and the measured responses for the two peaks are given in Fig. 6(b). The best-fit straight lines yield strain sensitivities of 1.074 and $1.075 \mathrm{pm} / \mu \varepsilon$ for the two peaks, respectively, a difference of less than $0.1 \%$.

The second dual-grating sensor was made in hydrogen-free $\mathrm{B} / \mathrm{Ge}$ fiber with the combination of
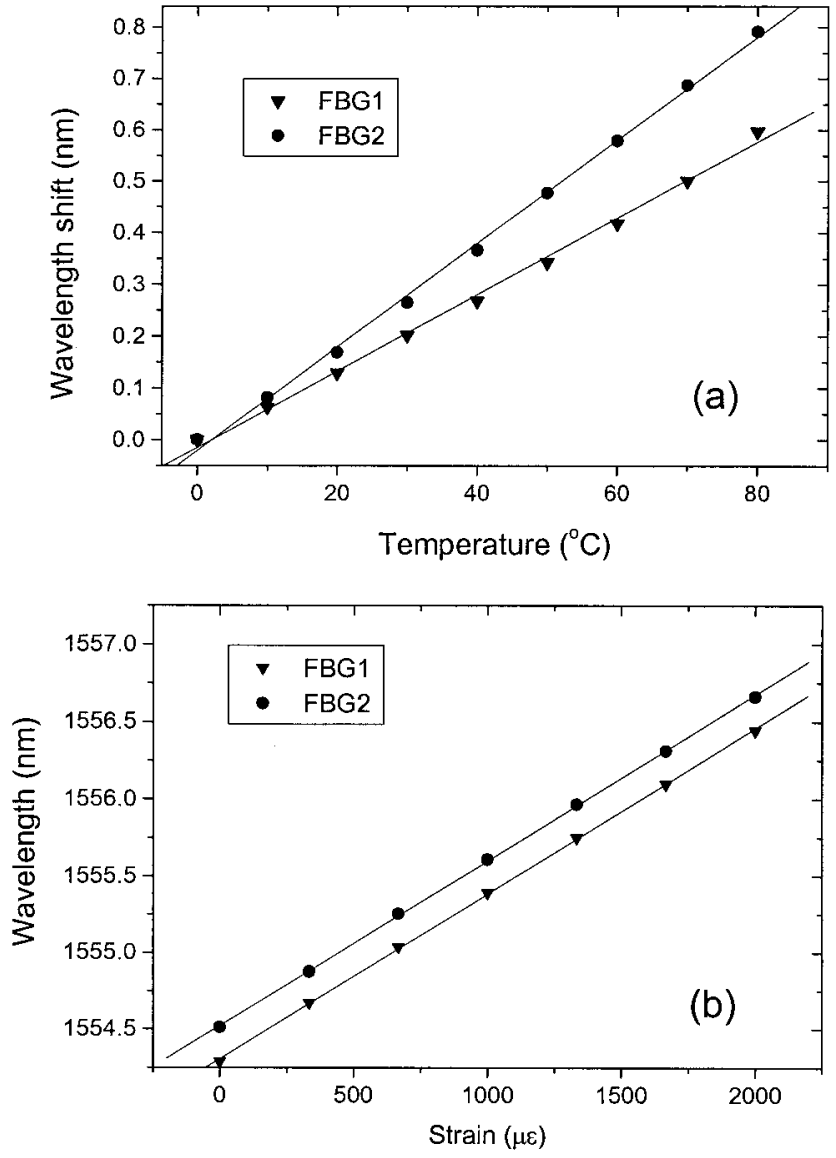

Fig. 6. Plots of wavelength shift for the first dual-grating sensor as a function of (a) temperature and (b) strain. FBG1, Type IA grating; FBG2, Type IIA grating.

Type I and Type IIA gratings. We stabilized the optical properties of the sensor by annealing at $120^{\circ} \mathrm{C}$ for $48 \mathrm{~h}$ before sensing measurement. The response of the second sensor to temperature is plotted in Fig. 7(a). From the best-fit line in Fig. 7(a), we obtain temperature coefficients of $10.20 \mathrm{pm} /{ }^{\circ} \mathrm{C}$ for FBG1 (Type IIA grating) and $9.56 \mathrm{pm} /{ }^{\circ} \mathrm{C}$ for $\mathrm{FBG} 2$ (Type I grating). The difference in temperature coefficient is $\sim 6.7 \%$, which is quite small compared with the first sensor and may not be efficient enough to discriminate the temperature and strain. Measured strain sensitivity response for the second sensor was plotted in Fig. 7(b). From the best-fit line in Fig. 7(b), we found that the two gratings have almost the same strain coefficient of $\sim 1.072 \mathrm{pm} / \mu \varepsilon$.

The third dual-grating sensor was made in hydrogenated B/Ge fiber with the combination of Type I and Type IA gratings. After the two gratings were inscribed as the structure of Fig. 4, the sensor annealed for $48 \mathrm{~h}$ at $120^{\circ} \mathrm{C}$ to stabilize the grating properties and remove residual hydrogen. The response of the third sensor to temperature is plotted in Fig. 8(a). From the best-fit line in Fig. 8(a), we obtain temperature coefficients of $7.53 \mathrm{pm} /{ }^{\circ} \mathrm{C}$ for FBG1 (Type IA grating) and $9.03 \mathrm{pm} /{ }^{\circ} \mathrm{C}$ for FBG2 (Type I grating). The difference in temperature coefficient 

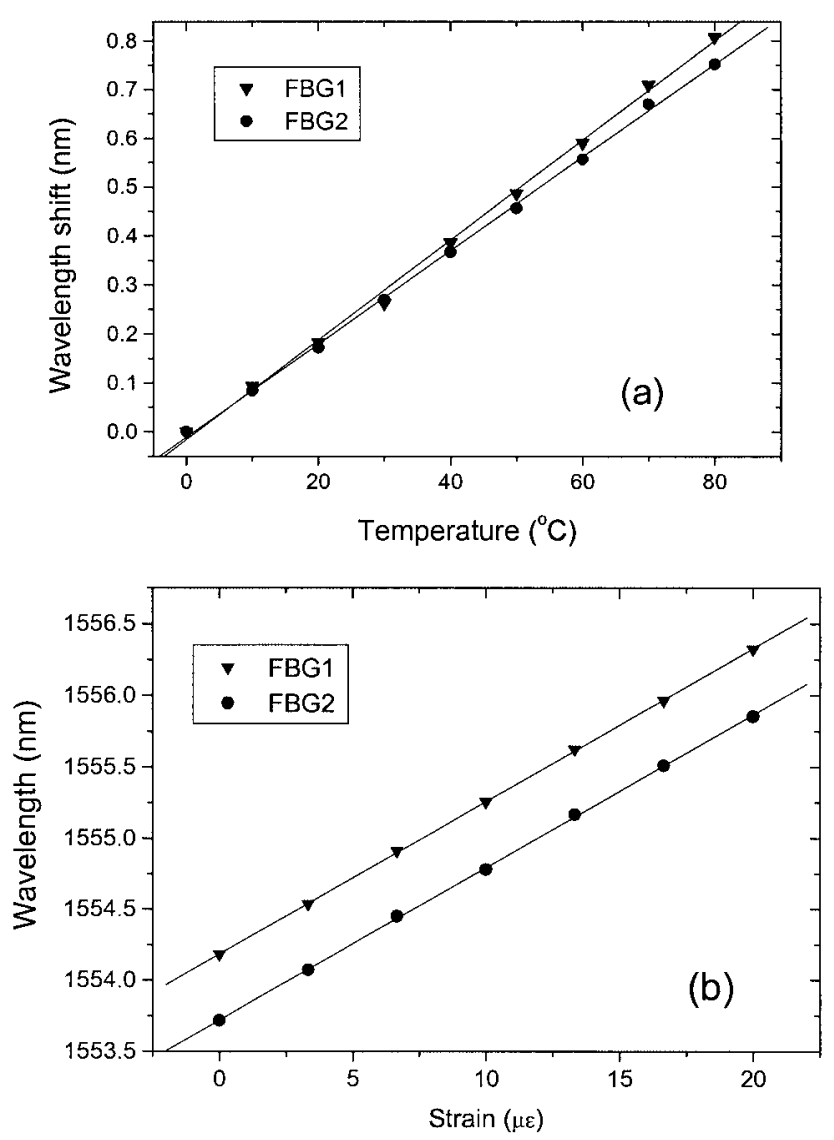

Fig. 7. Plots of wavelength shift for the second dual-grating sensor as a function of (a) temperature and (b) strain. FBG1, Type IIA grating; FBG2, Type 1 grating.

is $\sim 19.9 \%$, which is relatively small compared with the first sensor. Measured strain sensitivity response for the third sensor is plotted in Fig. 8(b). The best-fit straight lines yield strain very close to sensitivities of 1.067 and $1.068 \mathrm{pm} / \mu \varepsilon$ for the two gratings, respectively.

\section{Analysis of Errors in Dual-Grating Sensor Systems and Discussions}

Generally, for a dual-grating geometry, a sensitivity matrix

$$
\left[\begin{array}{l}
\Delta \lambda_{1} \\
\Delta \lambda_{2}
\end{array}\right]=\left[\begin{array}{ll}
\kappa_{T 1} & \kappa_{\varepsilon 1} \\
\kappa_{T 2} & \kappa_{\varepsilon 2}
\end{array}\right]\left[\begin{array}{c}
\Delta T \\
\Delta \varepsilon
\end{array}\right]
$$

can be constructed to provide for decoupling between the temperature and the strain information in signal processing. ${ }^{12}$ The matrix coefficients, $\kappa_{T i}, \kappa_{\varepsilon i}(i=1$, 2 ), are determined from independent measurements of the temperature and the strain sensitivities of the sensor. It is significant here that since the strain coefficients of the Type IA and Type IIA gratings are virtually identical, the signal processing required for decoupling in a sensor comprising two gratings of these types is significantly simplified. We expect, then, that a well-conditioned sensitivity matrix can be constructed for this sensor, facilitating its use in
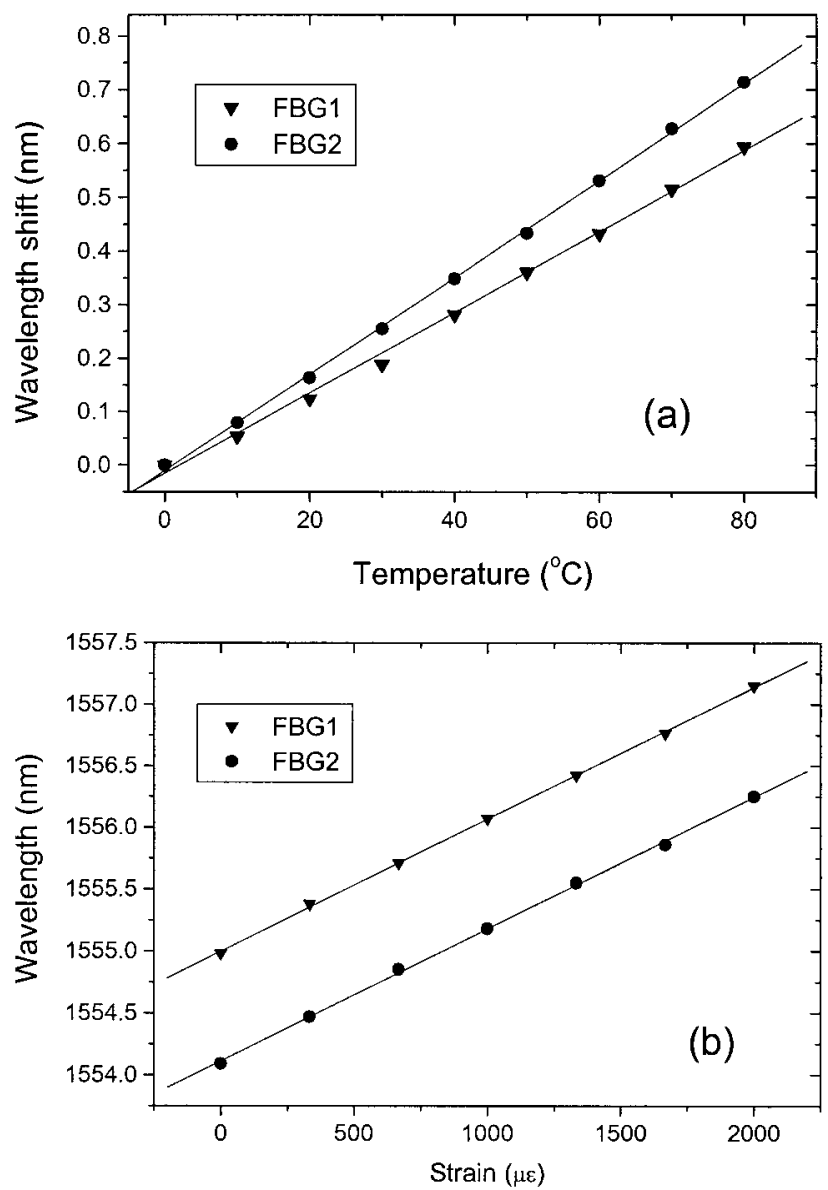

Fig. 8. Plots of wavelength shift for the third dual-grating sensor as a function of (a) temperature and (b) strain. FBG1: Type IA grating; FBG2, type I grating.

practical applications. From Eq. (1), one can easily decode the temperature and strain information as

$$
\left[\begin{array}{c}
\Delta T \\
\Delta \varepsilon
\end{array}\right]=\frac{1}{\kappa_{T 2}-\kappa_{T 1}}\left[\begin{array}{c}
\Delta \lambda_{2}-\Delta \lambda_{1} \\
\left(\kappa_{T 2} \Delta \lambda_{1}-\kappa_{T 1} \Delta \lambda_{2}\right) / \kappa_{\varepsilon 1}
\end{array}\right] .
$$

Assuming that the coefficients for temperature and strain are well known through careful measurement at each wavelength, one can use the following relationship to analyze the maximum error in temperature and strain for a dual-grating sensing system ${ }^{3}$ :

$$
\left[\begin{array}{c}
\Delta T_{\max } \\
\Delta \varepsilon_{\max }
\end{array}\right]=\left[\begin{array}{c} 
\pm\left(M_{T}-M_{T \varepsilon}^{2} / M_{\varepsilon}\right)^{-\frac{1}{2}} \\
\pm\left(M_{\varepsilon}-M_{T \varepsilon}^{2} / M_{T}{ }^{-\frac{1}{2}}\right.
\end{array}\right],
$$

where

$$
\begin{aligned}
M_{T} & =\frac{\kappa_{T 1}^{2}}{a^{2}}+\frac{\kappa_{T 2}^{2}}{b^{2}}, \\
M_{\varepsilon} & =\frac{\kappa_{\varepsilon 1}^{2}}{a^{2}}+\frac{\kappa_{\varepsilon 2}^{2}}{b^{2}}, \\
M_{T \varepsilon} & =\frac{\kappa_{T 1} \kappa_{\varepsilon 1}}{a^{2}}+\frac{\kappa_{T 2} \kappa_{\varepsilon 2}}{b^{2}},
\end{aligned}
$$

where $a$ and $b$ are the measurement errors for wavelengths $\lambda_{1}$ and $\lambda_{2}$, respectively. We expect, then, 
Table 2. Maximum Temperature and Strain Errors for Three Different Combinations in a Dual-Grating Sensing Scheme

\begin{tabular}{lcc}
\hline $\begin{array}{c}\text { Dual-Grating Sensing } \\
\text { Scheme }\end{array}$ & $\begin{array}{c}\text { Temperature } \\
\text { Errors } \\
\left({ }^{\circ} \mathrm{C} / \mathrm{pm}\right)\end{array}$ & $\begin{array}{c}\text { Strain Errors } \\
(\mu \varepsilon / \mathrm{pm})\end{array}$ \\
\hline Type IA-Type IIA & \pm 0.54 & \pm 4.4 \\
Type IIA-Type I (no H) & \pm 2.21 & \pm 20.4 \\
Type IA-Type I (with H) & \pm 0.94 & \pm 7.3 \\
\hline
\end{tabular}

that a well-conditioned sensitivity matrix can be constructed for this sensor, facilitating its use in practical applications. Adopting the error-analysis method described above, we calculated the temperature and strain errors for the above three different dual-grating sensors, and the results are listed in Table 2.

From Table 2 it is clearly known that the Type IA-Type IIA dual-grating sensor has the smallest errors in both temperature and strain compared with the other two sensors. The Type IIA-Type I (with hydrogen) dual-grating sensor has the largest errors, which is not efficient for practical use. It is also worth pointing out that the Type IA-Type IIA dualgrating sensor represents substantial improvements on the structures reported by Brady et al. $\left( \pm 1.72{ }^{\circ} \mathrm{C} /\right.$ $\mathrm{pm}$ and $\pm 17.4 \mu \varepsilon / \mathrm{pm})^{3}$ and by Xu et al. $\left( \pm 1.3^{\circ} \mathrm{C} / \mathrm{pm}\right.$ and $\pm 12 \mu \varepsilon / \mathrm{pm}) .^{2}$ Furthermore, in contrast with alternative structures, this sensor is readily extensible to wavelength multiplexed arrays in one or more fibers to form an effective, quasi-distributed sensing system with low splicing losses and high mechanical strength, thereby offering the advantages of singlelight source operation and robustness that elude most of the other configurations.

To optimize the proposed sensing scheme, we further investigate how the coefficient differences of strain and temperature influence the errors in the dual-grating sensor system. Figure 9 shows the calculated maximum temperature and strain errors as a function of $\kappa_{\mathrm{T} 1}$ in the dual-grating sensor system, assuming $\kappa_{T 2}=10.02 \mathrm{pm} /{ }^{\circ} \mathrm{C}, \kappa_{\varepsilon 1}=1.074 \mathrm{pm} / \mu \varepsilon$, and

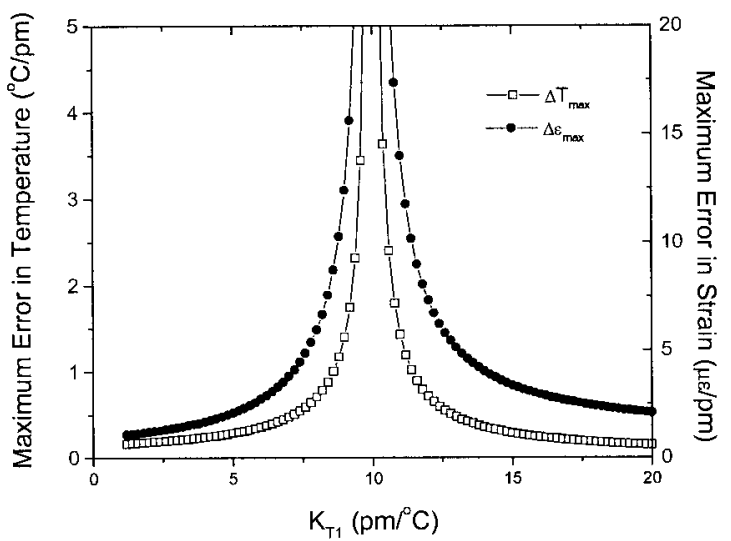

Fig. 9. Maximum temperature and strain errors as a function of $\kappa_{T 1}$ in the dual-grating sensor system, assuming $\kappa_{T 2}=10.02 \mathrm{pm} /$ ${ }^{\circ} \mathrm{C}, \kappa_{\varepsilon 1}=1.074 \mathrm{pm} / \mu \varepsilon$, and $\kappa_{\varepsilon 2}=1.075 \mathrm{pm} / \mu \varepsilon$.
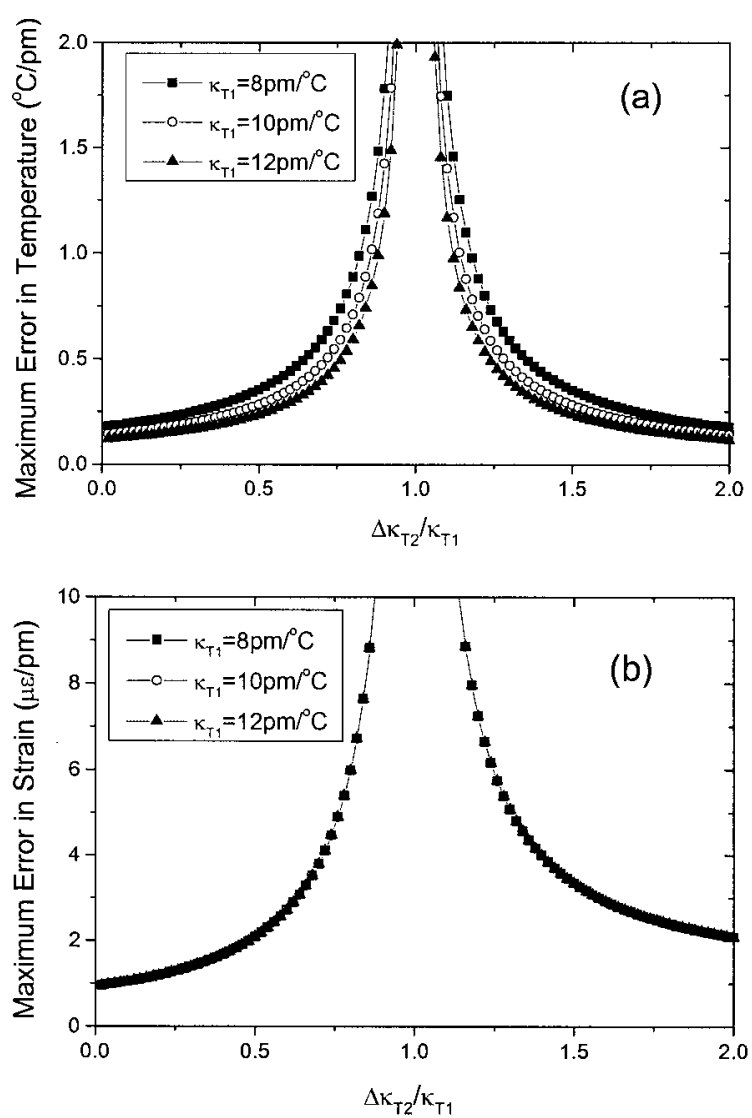

Fig. 10. (a) Maximum temperature errors and (b) strain errors as a function of the ratio of $\kappa_{T 2}$ and $\kappa_{T 1}$ in the dual-grating sensor system, assuming $\kappa_{T 1}=8,10$, and $12 \mathrm{pm} /{ }^{\circ} \mathrm{C}, \kappa_{\varepsilon 1}=1.074 \mathrm{pm} / \mu \varepsilon$, and $\kappa_{\varepsilon 2}=1.075 \mathrm{pm} / \mu \varepsilon$.

$\kappa_{\varepsilon 2}=1.075 \mathrm{pm} / \mu \varepsilon . \quad$ It is noted in Fig. 9 that, when the value of $\kappa_{T 1}$ is chosen to be close to that of $\kappa_{T 2}$, both the temperature and the strain errors increase rapidly. The reason is that the coefficient matrix in Eq. (1) tends toward a bad condition (i.e., $\kappa_{T 1} \kappa_{\varepsilon 2}-\kappa_{T 2} \kappa_{\varepsilon 1} \rightarrow 0$ ) if the value of $\kappa_{T 1}$ is close to that of $\kappa_{T 2}$. The larger difference between $\kappa_{T 1}$ and $\kappa_{T 2}$, the smaller errors in temperature and strain measurements, has been confirmed from the conducted experiments.

Figure 10 shows the calculated maximum temperature and strain errors as a function of the ratio of $\kappa_{T 2}$ and $\kappa_{T 1}$ in the dual-grating sensor system with different values of $\kappa_{T 1}$ assumed. It is interesting to see in Fig. 10(b) that the maximum strain error is dependent only on the ratio of $\kappa_{T 2}$ and $\kappa_{T 1}$, regardless of the value of $\kappa_{T 1}$. However, the maximum temperature error is dependent both on the ratio of $\kappa_{T 2}$ and $\kappa_{T 1}$ and on the value of the $\kappa_{T 1}$, as shown in Fig. 10(a). Figure 10(a) also shows that a larger value of $\kappa_{T 1}$ may lead to a smaller temperature error for the same ratio of $\kappa_{T 2}$ and $\kappa_{T 1}$.

\section{Conclusions}

This study has revealed that FBGs in B/Ge codoped fiber exhibit a strong dependence of temperature sensitivity on the grating type but weak dependence of the strain sensitivity. The grating temperature co- 
efficient is larger in hydrogen-free fiber than in hydrogenated fiber, with Type IIA gratings exhibiting the largest value overall, and a new, abnormal grating type, Type IA (which occurs in hydrogenated fiber), exhibiting the lowest value. The distinct sensitivity characteristics have been used to implement a novel, dual-grating sensor for the simultaneous measurement of temperature and strain, which exhibits performance superior to previously reported grating-based sensors for the same purpose.

This work was carried out with the support of the UK Engineering and Physical Sciences Research Council.

\section{References}

1. A. D. Kersey, M. A. Davis, H. J. Patrick, M. LeBlanc, K. P. Koo, C. G. Askins, M. A. Putnam, and E. J. Friebele, "Fiber grating sensor," J. Lightwave Technol. 15, 1442-1463 (1997).

2. M. G. Xu, J.-L. Archambault, L. Reekie, and J. P. Dakin, "Discrimination between strain and temperature effects using dual-wavelength fiber grating sensor," Electron. Lett. 30, 1085-1087 (1994).

3. G. P. Brady, K. Kalli, D. J. Webb, D. A. Jackson, L. Reekie, and J. L. Archambault, "Simultaneous measurement of strain and temperature using the first- and second-order diffraction wavelength of Bragg gratings," IEE Proc. Optoelectron. 44, 156-161 (1997).

4. H. J. Patrick, G. M. Williams, A. D. Kersey, J. P. Pedrazzani, and A. M. Vengsarkar, "Hybrid fiber Bragg grating/long period fiber grating sensor for strain/temperature discrimination," IEEE Photon. Technol. Lett. 8, 1223-1225 (1996).

5. S. E. Kanellopoulos, V. A. Handerek, and A. J. Rogers, "Simultaneous strain and temperature sensing with photogenerated in-fiber gratings," Opt. Lett. 20, 333-335 (1995).

6. S. W. James, M. L. Dockney, and R. P. Tatam, "Simultaneous independent temperature and strain measurement using infiber Bragg grating sensor,” Electron. Lett. 32, 1133-1134 (1996).

7. B. O. Guan, H. Y. Tam, S. L. Ho, W. H. Chung, and X. Y. Dong, "Simultaneous strain and temperature measurement using a single fiber Bragg grating," Electron. Lett. 36, 1018-1019 (2000).

8. Y. Liu, J. A. R. Williams, L. Zhang, and I. Bennion, "Abnormal spectral evolution of fiber Bragg gratings in hydrogenated fibers," Opt. Lett. 27, 583-585 (2002).

9. X. Shu, Y. Liu, D. Zhao, B. Gwandu, F. Floreani, L. Zhang, and I. Bennion, "Dependence of temperature and strain coefficients on fiber grating type and its application to simultaneous temperature and strain measurement," Opt. Lett. 27, 701-703 (2002).

10. W. X. Xie, P. Niay, P. Bernage, M. Douay, J. F. Bayon, T. Georges, M. Monerie, and B. Poumellec, "Experimental evidence of two types of photorefractive effects occurring during photoinscriptions of Bragg gratings within germanosilicate fibers," Opt. Commun. 104, 185-195 (1993).

11. L. Dong, W. F. Liu, and L. Reekie, "Negative-index gratings formed by a 193-nm excimer laser," Opt. Lett. 21, 2032-2034 (1996).

12. W. Jin, W. C. Michie, G. Thursby, M. Konstantaki, and B. Culshaw, "Simultaneous measurement of strain and temperature: error analysis," Opt. Eng. 36, 598-609 (1997). 